\title{
Corps, Arts et Spiritualité chez François Delsarte (1811-1871) : une dynamique d'éducation somatique expressive
}

\author{
Franck Waille* \\ E-mail: franck.cw@gmail.com
}

Cet article synthétise les recherches que Franck Waille a mené autour de François Delsarte de 2003 à 2013. Ces recherches ont pris une première forme avec la soutenance de la première thèse en français sur Delsarte en 2009 , centrée sur les interactions dynamiques entre le corps, les arts et la spiritualité chez Delsarte. Le dernier ouvrage de F. Waille sur la question met en valeur la dimension somatiques des enseignements expressifs delsartiens.

Mots clés

Delsarte. Éducation Somatique. Spiritualité.
Palavras-chave

Delsarte. Educação Somática. Espiritualidade.

\footnotetext{
* Franck Waille, enseignant dans le primaire et chercheur associé au Laboratoire de recherche historique Rhône-Alpes (LARHRA), est l'auteur de Corps, arts et spiritualité chez François Delsarte (1811-1871). Des interactions dynamiques (2009), premier doctorat en français concernant Delsarte. II donne des conférences et des cours de pratique corporelle expressive Delsarte au sein de la compagnie Chorâme créée en 2012. Il est l'auteur d'articles en français, anglais et portugais sur Delsarte, il a dirigé Trois décennies de recherche européenne sur François Delsarte (L'Harmattan, 2011) et codirigé François Delsarte, une recherhce sans fin (L'Harmattan, 2015). Son dernier ouvrage La méthode somatique expressive de François Delsarte. Histoire, esthétique, anthropologie : de la neurophysiologie à la métaphysique est publié par L'Entretemps en 2016. II a complété un postdoctorat au département de danse de l'Université du Québec à Montréal (2014-2015) et poursuit actuellement un autre postdoctorat à la Faculté d'Éducation de l'Universidade Federal do Rio Grande do Sul.
} 
François Delsarte, chanteur, professeur de chant et de déclamation français à la renommée d'abord européenne, laisse derrière lui un héritage riche, complexe et polyvalent d'une importance capitale pour les arts du spectacle vivant du XXe siècle. II « fut le premier à mener une recherche approfondie sur les mouvements, les gestes et les expressions du corps humain ${ }^{1}$, et l'« on s'entend généralement pour situer les débuts de la modernité des arts de la scène au moment [de ses] premières recherches et de [son] enseignement (...) sur le corps et son langage $»^{2}$. Ses enseignements engendrèrent une véritable Delsartemania posthume aux États-Unis dans les dernières décennies du XIXe siècle, et plus généralement, à travers les nombreux élèves de Delsarte, eurent une influence dans le monde entier parmi les pionniers du théâtre et de la danse moderne.

Dans le domaine de l'art dramatique (puis du cinéma), I'héritage de Delsarte est pour le moins contrasté. Certains le considèrent comme étant à l'origine d'une pratique artistique figée et monstrueusement mécanique ${ }^{3}$, alors que d'autres y voient le début de la modernité, en particulier par une prise en compte

1 Eugenio Barba, Nicola Savarese, L'Énergie qui danse. Dictionnaire d'anthropologie théâtrale, Montpellier, L'Entretemps, 2008, p. 167

2 André-G. Bourassa, "Danse au Québec et modernité ", Festival International de Nouvelle danse, 1987, p. 16.

3 Jacques Aumont, l'un des plus grands théoriciens du cinéma actuels, écrit ainsi : " L'œuvre de François Delsarte (...) propose une "méthode" fondée sur la physiognomonie, et ayant à sa base une sorte de dictionnaire ou de lexique, qui établit une correspondance entre des gestes et des états d'esprit : il existe une façon, et une seule, pour le corps, de manifester la colère, l'angoisse, la jalousie, l'inquiétude ou la tendresse. À toute émotion correspond un mouvement du corps ; inversement, un mouvement du corps représente adéquatement une émotion et une seule. Jouer, c'est, selon le système de la pantomime, apprendre à produire des "énoncés bien formés", dans un langage conventionnel et artificiel qui mobilise le corps. " (Jacques Aumont, Le cinéma et la mise en scène, Armand Colin, 2006, p. 20). nouvelle du corps dans le travail de l'acteur. II en va tout autrement pour la danse, globalement étrangère aux préoccupations directes de cet artiste, et pourtant domaine qui pourrait avoir le mieux reçu et développé son travail ${ }^{4}$. Par ailleurs, une des tendances de l'historiographie américaine est de réduire l'enseignement de Delsarte lui-même au seul chant et à une approche essentiellement théorique des réalités expressives, attribuant alors à ses héritiers américains l'essentiel des aspects pratiques d'un système expressif complexe car s'appuyant sur un cadre théorique très construit et relativement déconcertant de prime abord, car essentiellement structuré autour de concepts métaphysiques.

Cet héritage multiforme et contrasté oriente inévitablement le regard de l'historien vers l'enseignement d'origine, et cela dans une double perspective : essayer de faire le tri entre ce qui a été attribué à Delsarte et ce qu'il a vraiment enseigné, et comprendre les dynamiques internes de son travail en revenant sur les fondements historiques, biographiques, pratiques et théoriques de ce travail. Une difficulté méthodologique se fait immédiatement jour : l'ambition est de remonter à une pratique vivante essentiellement diffusée de manière orale, par une transmission directe de maître à disciples, mais dont il reste aujourd'hui des traces d'abord sous forme de documents écrits (diverses publications d'élèves), et de sources que nous pourrions appeler « semi-orales » :

4 « Si, comme le rapporte Nancy Lee Chalfa Ruyter (...), la lignée théâtrale de Delsarte a vu en son système "une formule rigide et mécanique pour l'expression", nous pouvons dire avec Odette Aslan que ce sont les danseurs qui "comprendront le mieux les idées de Delsarte et les débarrasseront de toute idée de pathos et de cliché." " (Christophe Damour, " L'influence de Delsarte sur le jeu de l'acteur aux États-Unis », in L'acteur de cinéma : approches pluridisciplinaires, Actes du colloque international tenu en octobre 2005 à Cerisy, Presses universitaires de Rennes, 2007, p. 20). 
les notes directes d'élèves prises durant les enseignements de Delsarte, et les propres notes de ce dernier (qui n'a jamais publié d'ouvrage concernant son travail). Cela implique une exigence méthodologique : tenter d'aborder les écrits en prenant en compte « la vie qui les féconde ${ }^{5}$ » selon une expression de l'artiste. Cela implique de situer tout document dans la visée esthétique générale de Delsarte, théorique comme pratique, et de l'aborder non seulement avec les outils de l'analyse scientifique intellectuelle, mais également avec ceux d'une pratique artistique, en particulier vocale et corporelle. Avant notre recherche, un seul travail scientifique s'était appuyé sur les manuscrits de Delsarte. ${ }^{6}$ II s'est agit de le prolonger, en nous centrant sur ce qui caractérise les enseignements expressifs de Delsarte : l'articulation des questions corporelles, spirituelles et artistique.

Notre thèse générale est que ces enseignements se sont centrés sur le travail du corps car le chant implique le corps comme instrument, et qu'il repose sur une pédagogie artistique visant à la liberté des élèves et non à la codification des expressions. Les travaux antérieurs au nôtre et les archives consultées nous ont permis de mettre en avant le fait que Delsarte, par ses expériences initiales - perte à 18 ans d'une voix prometteuse, auto-rééducation vocale, observations multiples des corrélations entre gestes et émotions -, a élaboré une méthode empirique d'observation des réalités expressives, qui reste le référent fonda-

5 Lettre de Delsarte à Mackaye du 9/10/1870 (conservée in The papers of the Mackaye Family, Baker Library, Special collections, Dartmouth College, Hanover, New Hampshire, box 22 , folder 3,document 3 ).

6 Elena Randi, L'estetica di François Delsarte: la dimensione spettacolare, thèse de doctorat en théorie et histoire de la représentation, université de Padoue, Padoue, 1992 teur de son travail. Ses observations l'ont amené à chercher à construire un cadre théorique d'explication des phénomènes observés, cela avant tout dans un but d'efficacité pédagogique. Travail pratique et réflexion esthétique l'ont amené à envisager réalités expressives, acte artistique et corps humain comme intimement associés à une dimension spirituelle et métaphysique, dans laquelle il affirme avoir trouvé le critère de compréhension des réalités expressives. Du fait de sa conversion au catholicisme, mais aussi des multiples milieux de formation qu'il a rencontrés (maçonnerie illuministe, ésotérisme chrétien, professeurs de latin et d'hébreux l'ayant, en particulier, introduit à la Kabbale), cette dimension spirituelle et métaphysique mêle théologie catholique et pensée ésotérique antique et médiévale. Ses enseignements proposent d'incarner les données métaphysiques utilisées, dans un travail expressif.

Par ailleurs, la dynamique pédagogique de Delsarte est directement inspirée de sa propre méthode empirique d'observation des réalités expressives, chez lui et autour de lui. Elle est synthétisée par cette phrase-clé :

«Un élève de ma méthode est un observateur silencieux des phénomènes qui l'entourent et se manifestent au-dedans de lui-même. " ${ }^{7}$

Cette dynamique pédagogique est annonciatrice de l'approche contemporaine appelée éducation somatique. Depuis la fin des années 1970, le terme « somatique » (de la racine grecque sôma, corps) cherche à échapper au dualisme âme/corps (du latin corpus) et fait référence à la perception de soi à la première

7 François Delsarte, in Alain Porte, François Delsarte, une anthologie, Paris, IPMC,1992, p. 260. 
personne dans le mouvement («je », et non «il/ elle »), à partir d'une perspective intégrée des différentes dimensions de l'individu. ${ }^{8}$ Tout ceci est au centre de l'approche delsartienne des réalités expressives.

\section{- Connaître le cadre théorique de Delsarte permet de mieux saisir son travail pratique}

La connaissance fine de son cadre théorique permet d'éviter certaines des incompréhensions et des déformations qui ont accompagnées la diffusion de ses enseignements. Ainsi, l'outil synthétique par excellence de Delsarte est-il ce qu'il a appelé " l'accord de neuvième ", qui lui servait à analyser n'importe quelle réalité, et en particulier les réalités expressives. Nous pouvons donner l'exemple suivant pour la main, publié par l'un de ses élèves, l'abbé Delaumosne (d'autres publications du même accord, par d'autres héritiers de Delsarte, présentent des tableaux sensiblement différents) :

8 Cf. Thomas Hanna, The Body of Life, New York, Alfred A. Knopf, \& "What is Somatics?», Somatics: Magazine-Journal of the Bodily Arts and Sciences, vol. V, no 4, Spring-Summer 1986, p. 4-8 (http://somatics.org/library/htl-wis1.html).
Figure 1: Accord de neuvième des mains, par Delaumosne

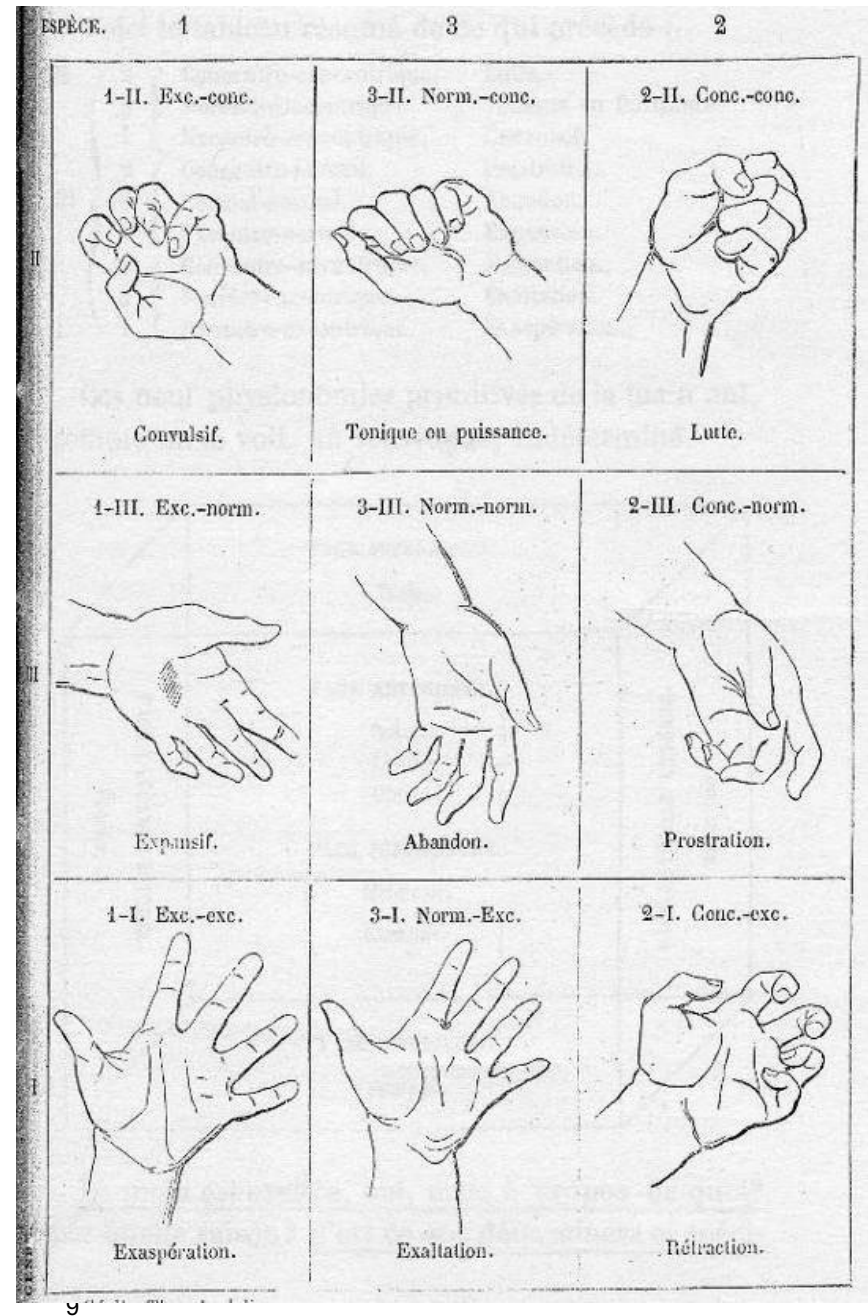

Delsarte, lors d'un cours en 1859, mettait en garde : «Où apprend-on la mimique ? Serait-ce dans le livre que Lebrun a intitulé Les Passions ${ }^{10}$ et où, donnant un type, il dit : voilà l'amour? Amour, substance cachée, visible seulement par les expressions sensitives qui correspondent au nombre illimité de transformations dont tu es susceptible et qui donne autant de physionomies différentes, voilà qu'ils t'ont réduit à un terme, voilà qu'ils l'ont mesuré, toi qui est l'infini ! »11. Or, c'est précisément

9 Abbé Delaumosne, Pratique de l'art oratoire de Delsarte, Albanel, Paris, 1874, p. 105.

10 Charles Le Brun, L'expression des Passions, 1698.

11 Esthétique appliquée, cours de F. Delsarte. Exposition en neuf leçons de l'art de l'orateur, du peintre et du musicien. Offert à M. Delsarte par son élève Alphonse Pages (Delsarte Collection, Hill Memorial Library, Louisiana State University, 
à ce type de réduction de son travail qu'ont pu conduire diverses publications faites par ses élèves ou par des personnes se réclamant de lui. Cette méprise fondamentale s'est en tout premier lieu fondée sur une incompréhension des tableaux d'accord de neuvième pour les différentes parties du corps, avec l'idée que Delsarte proposait des gestes codifiés pour telle ou telle émotion. ${ }^{12}$ La mésinterprétation de ce type de tableau vient largement de l'ignorance de son substrat métaphysique, et plus particulièrement théologique.

L'accord de neuvième est en effet basé sur la définition théologique de la Trinité (plus précisément sur la version qu'en a donnée Thomas d'Aquin, principale référence de Delsarte), débouchant sur la notion de circumincession. Chez Thomas d'Aquin, la relation est au centre de la Trinité : le Père est non seulement luimême, mais il est aussi relation avec le Fils, et relation avec le Saint-Esprit - ainsi en est-il pour chacune des deux autres personnes trinitaires. De la sorte, l'unité des trois personnes se manifeste en neuf termes. L'accord de neuvième est, pour Delsarte, la traduction plastique de la circumincession. La version de cet accord appliqué aux couleurs permet de saisir cela de manière simple. Voici le tableau que nous pouvons tirer des cours donnés par Delsarte (avec

Baton Rouge, Louisiane, États-Unis d'Amérique, box 12c, folder 40), cours théorique $n^{\circ} 1$.

12 Joseph Fahey, dans son article sur le delsartisme, écrit que " l'essentiel de [l'évaluation négative du système de Delsarte] est dû au pouvoir des images qui sont plus fortes que les mots. Les représentations graphiques de systèmes dérivés de Delsarte éclipsent fréquemment les aspects les plus subtils et complexes de ses écrits" ("Much of this negative assessment is due to the power of images over words. Pictorial representations of systems derived from Delsarte frequently overshadow the more subtle and complex aspects of his writing. » (Joseph Fahey, in.Th. Leabhart (dir.), Essays on François Delsarte, Mime Journal 2004/2005, Claremont, CA Pomona College Theatre Department for Claremont Colleges, 2005, p. 45). les limites de l'outil informatique utilisé ici):

Figure 2: Tableau de l'accord de neuvième des couleurs d'après les cours de 1859 (7e cours théorique)

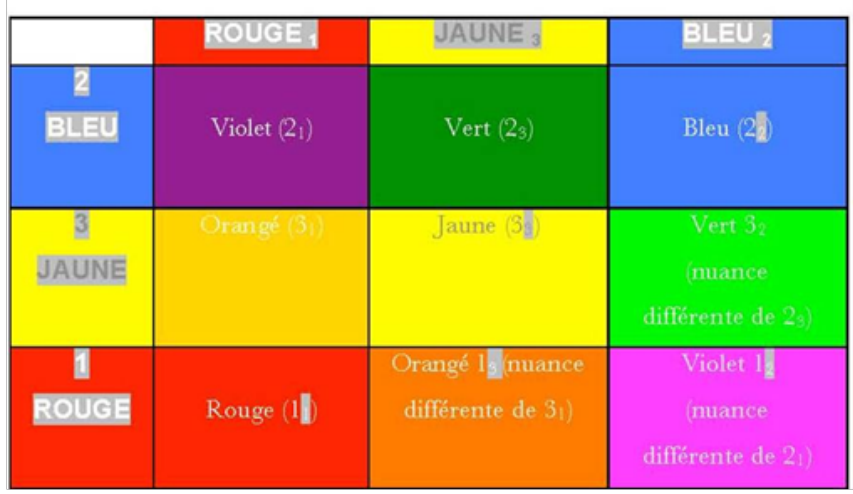

Nous avons les trois couleurs primaires comme critères horizontaux et verticaux de ce tableau à double entrée, puis dans la diagonale allant du rouge au bleu. Les six autres cases sont les combinaisons des trois couleurs primaires, où domine plutôt l'une ou l'autre. Cela forme la palette de base des couleurs. À chacune des couleurs primaires (ou éléments pouvant prendre leur place selon l'objet étudié), Delsarte a attribué une qualité particulière : excentrique (dynamique vers l'extérieur) pour le rouge, concentrique (dynamique opposée, vers l'intérieur) pour le bleu, neutre (équilibre des deux précédentes) pour le jaune. Cette qualité est directement tirée de la définition thomasienne de la Trinité (le Père est vu comme puissance fécondante, excentrique par excellence etc.), et donne une information de type énergétique sur ce qui est positionné dans telle ou telle case. Dans le même temps, les indications de type émotionnel indiquées sur le tableau de la main (« exaspération », « lutte », " abandon » par exemple), rappellent que les attitudes des différentes parties du corps qui sont alors mentionnées viendraient directement des observations de Delsarte, qui trouvent ici un cadre d'organisation et de présentation synthétique et dynamique : «ce 
moyen si simple [lui permet] d'inscrire les faits nombreux qu['il a] poursuivis, et qui s'élèvent à des millions, [sans qu'il se perde] dans ces millions de faits ${ }^{13}$. L'accord de neuvième, structuré selon des règles précises, est en même temps assez souple pour épouser les infinies variations des « motivations intérieures », des émotions. Dans une lecture erronée de ce tableau, on omet sa dynamique interne liée à son aspect synthétique, qui n'est pas normatif. Ce tableau est avant tout un outil présentant un processus procédant par subdivisions successives : chaque élément, issu du passage de trois genres à neuf espèces, peut être à son tour divisé selon la même logique ternaire selon un processus sans fin (les espèces pouvant à leur tour être combinées avec les trois genres, c'est-à-dire chacune divisée par trois etc.). Ce processus de démultiplication lui permet d'affiner son analyse pour détailler les phénomènes expressifs, poussant la description des phénomènes dans la limite de l'applicable ${ }^{14}$. Par ailleurs, chacun de ces éléments, se rapportant à une partie précise du corps, doit être combiné avec ceux des autres parties du corps, toute expression est faite de la participation de différents éléments corporels, voire du corps entier. L'ensemble de ces combinaisons est alors difficilement dénombrable. La démarche indiquée ouvre ainsi à l'infini le champ des possibles de l'analyse et du travail de l'expression humaine. Delsarte a donc envisagé un travail artistique qui, loin d'être figé, tend vers une palette illimitée d'expressions. Avec l'accord de neuvième,

13 Cours de M. Delsarte aux Sociétés savantes, (Delsarte Collection, op. cit., box 12 b, folder 54), cours $n^{\circ} 6$.

14 Delsarte mentionnait des milliers, voire des millions d'attitudes possibles pour telle ou telle partie du corps. II liste par ailleurs 28149 " signes inflexibles. Ces 28149 formes combinées entre elles produiront des millions de modifications, qui sont certes bien dans la nature. " (Fusion des signes, in Fonds Serge Bouts, Parisn carton jaune, dossier 13, document 6). il dispose d'une grille de lecture du monde, et en particulier des phénomènes expressifs qui tendent par leur nombre vers l'infini, et qu'il prétend ramener à neuf. Non pour réduire tout à neuf, mais pour pouvoir tout travailler à partir de neuf éléments. Nous pouvons voir l'outil qu'est l'accord de neuvième comme une tentative de dépasser les limites imposées par les moyens techniques de représentation du corps en mouvement avant l'invention du cinématographe : il propose de s'entraîner à enchaîner les différentes attitudes indiquées « de toutes les manières ${ }^{15}$ (c'est-à-dire dans n'importe quel ordre) comme le précise Alfred Giraudet, un élève de Delsarte qui enseigna lui-même. Et, de la sorte, de faire l'expérience de différentes attitudes, positions et niveaux de tensions musculaires possibles de telle ou telle partie du corps.

Sans son substrat métaphysique, ce tableau est donc un outil figé et normatif, alors qu'il est essentiellement dynamique. Notre travail est le premier a avoir mis en relation la théologie thomasienne et les outils théoriques de Delsarte, ce qui permet ainsi de les bien comprendre.

\section{—Une pédagogie cherchant à coller aux réalités expressives humaines}

Les accords de neuvième pour les différentes parties du corps étaient donc des supports pédagogiques pour un enseignement corporel expressif permettant de travailler de manière précise et fine les différents éléments constitutifs de l'expression gestuelle. La réalité vivante et dynamique de l'accord de neuvième est au cœur de la pédagogie artistique de Delsarte.

15 Alfred Giraudet, Mimique, Physionomie et Gestes. Méthode Pratique D'après le système de François Del Sarte pour servir à l'expression des sentiments, Paris, Ancienne Maison Quantin / Librairies-Imprimeries Réunies, 1895, p. 119. 
Cette pédagogie est en effet centrée sur une observation constante des réalités expressives, tout en ayant un objectif clairement affirmé : la spiritualisation de l'artiste afin d'aider à la spiritualisation du spectateur. Pratiquement, elle vise à rendre l'élève autonome vis-à-vis de l'enseignant, en lui proposant une série de règles à vérifier par lui-même, ce qui lui permet de ne pas dépendre de la subjectivité du professeur mais de pouvoir justifier ses choix en fonction de lois confortées par l'observation de soi et des autres.

Le parcours de Delsarte, qui découvrit par ses propres observations l'essentiel de ce qu'il a transmis et qui souffrit de l'arbitraire de professeurs au conservatoire, puis qui se convertit au christianisme, est le modèle de cette démarche pédagogique. Ce parcours trouve un écho dans les expériences d'Augustin d'Hippone, relatées en particulier dans Les confessions, auxquelles Delsarte se réfère explicitement : «Et bien, le spectacle que je veux vous faire contempler est tout intérieur. (...) Descendre en vous-même : saint Augustin » 16 écrit-il. L'idée directrice est qu'en se connaissant soi-même, il serait possible de découvrir que nous serions habités du divin. Éveiller la conscience de l'être humain comme porteur du divin est l'objectif que Delsarte fixe à l'acte artistique. L'artiste, selon lui, doit être le moyen de cet éveil, et pour cela il doit lui-même être éveillé.

Dans le même temps, Delsarte se singularise vis-à-vis de la tradition augustinienne, qui considérait que l'image du divin concernait l'âme - le corps étant conçu comme contenant. Delsarte écrit lui : “ C'est à cette mystérieuse exploration de vous-même, c'est à ce

16 L'homme objet d'étude (Delsarte Collection, op. cit., box 1a, folder OS 36b, document 18), p. 1. voyage au travers de vous-mêmes que je vous convie. Jusque dans les profondeurs de votre organisme [sont rayés : être, nature] je veux vous faire descendre. J'y guiderai vos pas, et là, du sein même des obscurités jailliront à vos yeux les lumières translumineuses de la Jérusalem que nous portons au-dedans de nous. ${ }^{17}$ Après avoir pensé à des mots plus abstraits et philosophiques, Delsarte en revient, avec le terme d'organisme, à celui qui se rapproche le plus de son expérience personnelle d'artiste, mais aussi d'une expérience mystique associée à sa conversion, dans laquelle il affirme avoir vu que « que le corps humain est l'alphabet (...), l'encyclopédie du monde (...) et le Diamant de la création $^{18}$ - en d'autres termes, le corps serait l'objet à étudier de manière préférentielle pour toute compréhension des phénomènes expressifs. Cette étude concerne tant le corps dans son organisation que dans son fonctionnement, d'où les formations que Delsarte suivit en anatomie et en physiologie. Par l'idée d' " encyclopédie du monde », il faut comprendre le corps comme reflet du divin manifesté dans l'univers entier. Cela permet de préciser la perspective générale de Delsarte : " restitu[er] à la métaphysique les bases physiques sans lesquelles elle n'est qu'une ombre vaine $»^{19}$ selon lui.

Le substrat spiritualiste de ses enseignements n'empêche pas qu'ils puissent avoir une « apparente laïcité absolue »20. Ted Shawn, ar-

\section{Ibid}

18 Delsarte Collection, op. cit., box 1, folder $36 \mathrm{~b} /$ items $1-7$, document 7 .

19 Notre méthode (The papers of the Mackaye Family, op. cit., box 22 ML5 (22), folder 1), document 4.

20 « L'apparente laicità assoluta del metodo (...) » (Roberto Tessari, "L'attore in Delsarte », in Elena Randi, François Delsarte : le leggi del teatro. II pensiero scenico del precursore della danza moderna, Roma, Bulzoni editore, 1993, p. 83). 
dent défenseur du travail de l'artiste français et premier chorégraphe moderne aux États-Unis, affirme ainsi, d'après son expérience : « que l'on adhère ou non à la métaphysique de Delsarte, son système est utilisable (...) $»^{21}$. La capacité de Delsarte à traduire en termes concrets des données métaphysiques est directement liée à l'importance centrale du corps chez lui, et c'est dans le cadre de l'analyse et du travail du corps qu'est le mieux saisie l'articulation profonde entre les aspects théoriques et pratiques de ses enseignements. Cette importance du corps humain se retrouve en particulier à deux niveaux : dans l'analyse du mouvement (du corps en mouvement), et dans la conception générale de l'être humain, non dualiste.

Une analyse du mouvement à la jonction des observations et du cadre théorique de Delsarte

L'analyse du mouvement faite par Delsarte est bâtie autour de trois éléments centraux : la notion d'opposition, l'importance du haut du buste (la région du thorax) et le rôle du poids. Ces trois éléments renvoient aux différentes sources du système expressif delsartien.

La notion d'oppositions est considérée comme la source de l'équilibre statique et dynamique, et permettrait par là même le mouvement : Delsarte parle alors de la loi « des oppositions dans la dynamique ${ }^{22}$. Les observations concernant la marche, illustrées par exemple à la fin du XIXe siècle par les chronophotogra-

21 Ted Shawn, Chaque petit mouvement. À propos de François Delsarte, Paris, Éditions complexe/Centre National de la Danse, 2005, p. 63.

22 Notebook of Mackaye while studying with Delsarte (Delsarte Collection, op. cit., range 35), p. 91. phies d'Étienne-Jules Marey, montrent que celle-ci se fait selon un mouvement d'opposition controlatéral : si la jambe droite est avancée, c'est que l'épaule gauche l'est également (alors que jambe gauche et épaule droite sont en arrière). Cette notion d'opposition a également été repérée par Delsarte dans la statuaire grecque antique, qu'il étudia de nombreuses années au Louvre, et où il affirme avoir découvert les règles de l'équilibre du corps et des lois de l'expression humaine par le geste et le mouvement. La statuaire grecque a introduit la position en contrapposto « qui se caractérise par ce décalage latéral de la hanche provoqué par l'appui d'équilibre sur un seul pied et le déplacement latéral de la tête engendré par la torsion du buste. (...) Une combinaison de mouvement et de repos, d'équilibre et d'asymétrie : une danse des oppositions ${ }^{23}$ qui permet de donner une sensation de mouvement à des figures figées dans le marbre. Enfin, d'un point de vue théorique, la notion d'opposition est commune à la théologie trinitaire et l'ésotérisme. Ainsi, la notion d'oppositions complémentaires, ou, seIon les mots d'Antoine Faivre, " de "dualitudes" " ou d'“ antagonismes structuraux "24 ayant une dynamique d'unité (oppositions haut/bas, spirituel/matériel, homme/femme, etc.) , est « présente partout dans l'hermétisme ${ }^{25}$. Les oppositions sont alors considérées comme la principale structure du monde, « tout (...) pren[ant] place dans un ensemble de forces opposées en vivante tension ${ }^{26}$. Delsarte li-

23 Eugenio Barba, Nicola Savarese,, L'Énergie qui danse. Dictionnaire d'anthropologie théâtrale, Montpellier, L'Entretemps, 2008, p. 170

24 Antoine Faivre, Accès de l'ésotérisme occidental, Paris, Gallimard, 1996, I, p. 44.

$25 \mathrm{lbid}$.

26 Ibid., I, p. 45. 
sait ces antagonismes structuraux comme sous-tendant l'ensemble de la révélation chrétienne et parlait alors $d^{\prime}$ ' antinomies $»^{27}$. Plus théologiquement, le principe d'opposition est au cœur de la définition thomasienne de la Trinité : il est alors à l'origine de l'individuation et donc de la relation entre les trois personnes trinitaires. En effet, dans la mesure où les relations définissent les personnes divines chez l'Aquinate, ce sont des face-à-face, c'est-àdire des relations d'opposition. ${ }^{28}$

L'importance centrale du torse est également au croisement des observations et des théories de Delsarte sur la symbolique du corps humain. Si nous reprenons l'exemple de la marche, l'analyse delsartienne montre qu'elle n'a sa source ni dans les jambes ni dans le bassin, mais bien dans le haut du torse. Une analyse actuelle indique que « quand une marche est une succession de chutes en avant [ce qui est la cas de la marche la plus courante], la ligne figurant l'axe du corps - un axe qui passerait de l'oreille à la cheville - est en inclinaison permanente vers l'avant par rapport à une ligne d'aplomb verticale. Les jambes doivent rattraper rapidement l'équilibre et amortir un supplément de poids dû à la chute. ${ }^{29}$

Plus généralement, Delsarte considérait que « tout mouvement provient de l'impul-

27 « Antinomies. Quoi ! Un tel renversement !!! L'incréé va naître et souffrir. L'immortel va recevoir la mort. Le verbe va se faire chair (...). » (Les folies de la sagesse divine, Delsarte Collection, box 1a, Folder OS 36b/item 6, document 1).

28 Thomas d'Aquin, Somme théologique, la, q. XL, a. 3, Paris, Cerf, 1984, vol. 1, p. 435-437.

29 Claire Hegen, Yves Marc, "La marche et l'acteur ", in Odette Aslan (dir.), Le corps en jeu, op. cit, p. 361. Ces auteurs présentent également d'autres types de marches : par une suite de chutes sur la verticale, par traction (le corps tracte le corps qui reste à la vertical ou s'incline vers l'arrière), par propulsion (tronc vertical propulsé vers l'avant par la jambe arrière). sion du cœur ${ }^{30}$, cœur qu'il faut entendre à la fois comme l'amour et le cœur physique. En d'autres termes, la poitrine est vue comme le principal centre dynamique du corps : c'est d'elle que procèderait le mouvement expressif. Dans la symbolique corporelle qu'il a développée sur une base trinitaire, il voyait le haut du torse, et plus spécifiquement encore la région du cœur et du plexus solaire, comme la partie la plus spirituelle et la plus émotionnelle du corps, par opposition aux parties plus vitales (le bassin, les jambes) ou plus intellectuelle (la tête).

Cette impulsion du cœur dans le mouvement est associée à l'importance du poids, ce qui est toujours lié à des observations et à son cadre théorique. Delsarte fait en particulier une série d'associations à partir de notions bibliques (mesure, nombre et poids ${ }^{31}$ ) et d'analyse rationnelle des phénomènes sur le monde trinitaire (temps, espace, mouvement ${ }^{32}$ ), qui l'amène a énoncer que « le poids engendre le mouvement ! " ${ }^{33}$ Cette mise en lien du poids et du mouvement est capitale car elle induit une notion de qualité du mouvement : non pas fait en force, à coup de tensions musculaires, mais par la gestion fluide du poids dans une série d'équilibres et de déséquilibres. Cette notion, avec l'importance de l'impulsion dans le torse, est fondamentale dans l'histoire du développement de la danse moderne.

30 Carnet de Delsarte (Delsarte Collection, op. cit., box 2c, folder 88).

31 « Mais tu as tout réglé avec mesure, nombre et poids », (Sg 11, 20, in La Bible de Jérusalem, Paris, Cerf, 1994, p. 975).

32 Notebook of Mackaye while studying with Delsarte (Delsarte Collection, op. cit., , box 2c, folder 2), p. 115.

33 Cours de M. Delsarte aux Sociétés savantes $n^{\circ} 5$, in Alain Porte, François Delsarte, ..., op. cit., p. 109. 
La conception delsartienne de l'être humain et des processus émotionnels a des échos actuels

La notion de qualité du mouvement est également dictée par une idée directrice simple et centrale chez Delsarte : l'expression - en particulier par le mouvement - ne doit pas être dictéepar un soucisde virtuosité, mais chercher à refléter une réalité intérieure. Cette dynamique expressive repose sur une conception non dualiste de l'être humain.

L'importance du corps place Delsarte dans une position singulière vis-à-vis du christianisme de son époque, durant laquelle «le chrétien est pressé de mépriser son corps ${ }^{34}$. Mais cette place du corps est à comprendre dans le cadre d'une vision unifiée de l'être humain, dans laquelle âme (ou principe spirituel) et corps sont intimement associés. Les expériences initiales de Delsarte en tant qu'artiste, observant en permanence des corrélations entre réalités émotionnelles et processus expressifs, ont trouvé deux types d'appui théoriques. D'une part, Delsarte a pu faire une lecture juste de l'anthropologie thomasienne, loin des déformations du thomisme dominant à son époque pour l'interprétation des textes de l'Aquinate. Cela sans doute grâce à son intuition, mais aussi à une solide formation auprès de jésuites (l'abbé de Ravignan en particulier) et de dominicains (il fut I'un des premiers membres du tiers ordre dominicain rétabli en France par Lacordaire en 1844), et une lecture directe du texte d'origine. II citait ou reformulait de manière systématique, dès lors qu'il parlait de sa conception de l'être humain, une phrase de Thomas d'Aquin : "L'âme, considérée selon son essence, est la forme du corps.

34 Gérard Cholvy, Yves-Marie Hilaire, Histoire religieuse de la France contemporaine. 1800-1880, Paris, Privat, 2000, p. 60.
„5'Aquinate s'inscrivait dans la vision aristotélicienne de l'homme, dans laquelle la forme est le principe non matériel de l'existence des choses, permettant que chaque type de chose soit le genre de chose qu'elle est. L'âme, en tant que forme du corps, lui est alors substantiellement unie. Cette conception est donc non dualiste et insiste sur l'unité complexe du composé humain réunissant deux principes opposés : l'âme, cause formelle, et le corps, cause matérielle. C'est insister sur l'unité fondamentale de l'être humain dans toutes ses dimensions.

D'autre part, Delsarte s'appuie sur la notion de correspondances universelles utilisée dans les milieux ésotériques (« Ce qui est en bas, est comme ce qui est en haut ${ }^{36}$ ), dont il fait une reformulation pour l'appliquer à l'être humain, reformulation que nous avons proposé d'appeler « loi des correspondances humaines » et qui est la suivante : " à chaque fonction spirituelle répond une fonction du corps ; à chaque grande fonction du corps répond un acte spirituel $»^{37}$. Cette dynamique est directement celle qui est à l'œuvre dans la recherche d'une expression qui soit reflet de l'âme, et dans l'idée que l'acte artistique pourrait agir sur l'intériorité du spectateur. Elle a trouvé de très nombreux échos, tant dans le théâtre contemporain que dans la danse moderne. Ainsi, l'idée que le corps en mouvement dirait quelque chose de l'âme ne devint pas seulement « un axiome de la danse moderne américaine ${ }^{38}$, mais de la danse mo-

35 Thomas d'Aquin, Somme théologique, la, Q. 76, a.1, sol. 4, op. cit., vol. 1, p. 665.

36 La Table d'Émeraude et sa tradition alchimique (attribué au légendaire Hermès Trismégiste), présenté par Didier Kahn, Paris, Les Belles Lettres, 2002, p. 43.

37 Delsarte Collection, op. cit., box 1, folder 36a/items 9-14, document 3.

38 Nancy Lee Chalfa Ruyter, in Ted Shawn, Chaque petit mouvement..., op. cit., p. 18. 
derne dans son ensemble, des deux côtés de l'Atlantique.

Delsarte ne se contente pas d'utiliser ses référents théologiques et ésotériques, il leur donne aussi un prolongement inédit. En effet, l'association de ce cadre théorique et d'une pratique artistique est novatrice vis-à-vis des référents utilisés, et a donné, pour la première fois à notre connaissance, un prolongement corporel et expressif à des données anthropologiques appuyées sur la philosophie, la théologie et la pensée ésotérique. Par ailleurs, les conclusions de son analyse des processus émotionnels basée sur l'unité fondamentale de l'être humain, trouvent de multiples échos dans les recherches actuelles en physiologie de l'action et de l'émotion. Les travaux d'Alain Berthoz et d'Antonio Damasio, en particulier, insistent sur l'association intime des stimuli intérieurs (les émotions) et de leurs manifestations (les expressions). Ils se positionnent par conséquent dans une perspective absolument opposée au dualisme issu de la modernité occidentale, ce qui était précisément le cas de Delsarte à son époque, même si les outils utilisés par les uns et les autres sont différents.

\section{— L'approche symbolique du corps par Delsarte : une piste pour éclairer les liens entre arts et spiritualité}

L'importance du torse selon Delsarte dans tout mouvement, et en particulier dans tout mouvement expressif, telle que nous l'avons présentée plus haut, est incluse dans une approche symbolique globale du corps. Celle-ci se déploie en deux temps. Delsarte appréhendait le corps par une série de subdivisions ternaires ayant chacune une dimension énergétique, ex- pressive et directionnelle. La plus globale de ces divisions, qu'il appelait la division essentielle, individualise bassin (vital, porté vers l'extérieur, l'avant, excentrique), tête (intellectuelle, portée vers l'intérieur, concentrique) et le thorax (spirituel et émotionnel, faisant l'équilibre du bassin et de la tête, contenant le cœur, les poumons et le plexus solaire) : elle concerne donc les foyers d'activité organique. Cette division peut inclure les membres comme prolongements. La seconde grande division est appelée par Delsarte division esthétique : elle est en quelque sorte la projection expressive de la division essentielle. Elle connaît une série de subdivisions, qui viennent préciser les dynamiques expressives du corps. Voici les représentations possibles de ces deux approches du corps :

Figure 4: Division essentielle du corps (le deuxième dessin intègre les membres)

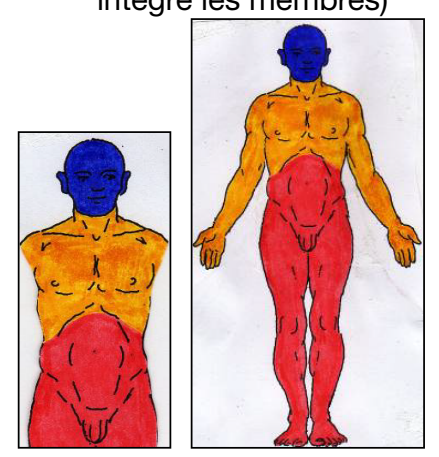

Figure 5: Division esthétique du corps et sa première subdivision

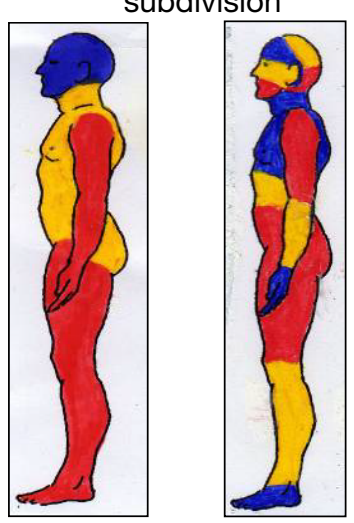

Dans ces représentations que nous avons faites à partir des informations contenues dans 
les documents d'archives, la couleur rouge caractérise ce qui est vital, le bleu ce qui est intellectif, le jaune ce qui est spirituel et émotionnel. C'est sur ce dernier point que nous voulons conclure ici. Nous voyons que les parties du corps plus spécifiquement associées aux dynamiques spirituelles et émotionnelles sont le torse dans son ensemble (source du mouvement) - division esthétique -, et plus spécifiquement le haut du torse (division essentielle) ou la région que Delsarte appelait épigastrique (zone de l'estomac), qui est celle du plexus solaire (première subdivision de la division essentielle). Ces approches symboliques du corps sont, d'une certaine manière, la synthèse des observations de Delsarte et de son cadre théorique métaphysico-spirituel. Elles mettent en valeur les principales dynamiques de ses enseignements tant pratiques que théoriques, en en particulier celle-ci : selon lui, tout travail de type expressif, orienté vers le développement de la dimension émotionnelle de l'être humain, est indissociablement lié à un travail spirituel se faisant de facto, en dehors de tout cadre religieux éventuel (mais sans contradiction avec celui-ci). Et le travail du corps et du mouvement tel qu'il les propose, en favorisant le rôle du torse, agiraient directement au plan psycho-spirituel en favorisant la spiritualisation de l'artiste et, par écho, de son travail.

Cette approche nous semble pouvoir aujourd'hui encore susciter de l'intérêt et mériter l'expérimentation. C'est le cas de l'ensemble des enseignements pratique de Delsarte nous semble-t-il.

\section{Actualité potentielle des} enseignements expressifs de Delsarte
Notre travail examine minutieusement les éléments de débat sur la réalité et le contenu des enseignements corporels de Delsarte. Cela nous permet d'affirmer que les conceptions précises du corps, de l'être humain dans son ensemble et du mouvement, sont associées chez lui à un travail corporel minutieux, en cohérence avec le fonctionnement général de ses enseignements : toujours associer théorie et pratique, car selon lui la théorie est " intéressant(e) comme moyen d'abréger le chemin, mais il faut arriver à la pratique ${ }^{39}$. Nous présentons des éléments permettant de mieux connaître l'entraînement corporel élaboré par Delsarte, conçu dans le cadre d'une pédagogie du chant et de la parole articulée, fondamentalement orientée vers le geste et le mouvement.

Une connaissance précise des enseignements de Delsarte, prenant en compte leur complexité et leur finesse, permet de les envisager comme toujours viables et pertinents dans la perspective de la formation des artistes du spectacle vivant. Les conceptions delsartiennes de l'acte artistique comme étant intimement lié aux réalités émotionnelles observées, l'insistance de Delsarte sur ce que nous appelons aujourd'hui la communication non-verbale et sur le travail corporel, intéressent toujours le travail du danseur ou du comédien. Ainsi, il y a une réévaluation positive de ces enseignements dans le monde du théâtre américain depuis les années 1960, d'un point de vue historique mais également pour une pratique contemporaine. ${ }^{40}$ Une série de travaux déve-

39 Cours de M. Delsarte aux Sociétés savantes, op. cit., $\mathrm{n}^{\circ} 10$.

40 Cf. les articles suivants : Kirby E. T., « The Delsarte Method: 3 Frontiers of Actor Training ", The Drama Review, volume 16, number 1 (T.53), mars 1972, p. 64-67; Gautam Dasgupta, "Commedia Delsarte ", Performing Arts journal, $\mathrm{n}^{\circ}$ 45, Baltimore, Maryland, USA, septembre 1993, vol. XV, $n^{\circ}$ 3, p. 95-102; Joseph Fahey, « Quiet Victory : The Professional Identity American Women Forged Through Delsartism », 
loppent l'idée qu'il y aurait eu une déviation vis-à-vis des enseignements d'origine ${ }^{41}$, et que ce sont ces déviations qui dévalorisèrent le travail de Delsarte en Amérique. En ce sens, la recherche approfondie que nous avons effectuée permet de mieux saisir les dynamiques du travail de Delsarte et pourrait intéresser directement les milieux de la formation du comédien et de l'acteur. Étrangère à toute notion de codification, la pédagogie de Delsarte est axée sur l'observation des réalités expressives et un travail corporel rigoureux de désapprentissage et de réapprentissage. II ambitionne l'autonomie de l'apprenant et sa liberté créative.

Le travail de préparation corporelle, en particulier, garde son intérêt dans ce qu'Eugenio Barba appellele travail pré-expressif. Le point de départ de ce travail, que Delsarte appelait la décomposition - qui correspond globalement à la décontraction, mais en mettant l'accent sur le relâchement des différents segments corporels à partir des articulations - fut sans doute une innovation en Occident concernant le travail corporel à visée expressive. Dans le processus de décomposition, l'utilisation du poids joue un rôle central, comme dans tout ce qui concerne le corps en mouvement chez Delsarte. Revenir à cet enseignement fondateur est une occasion d'enrichir les pratiques actuelles, qui ont pu indirectement s'en inspirer, tout en s'éloignant de certains des proces-

Thomas Leabhart (dir.), Essays on François Delsarte, Mime Journal 2004/2005, Claremont, Californie, Pomona College Theatre Department for Claremont Colleges, 2005, p. 43-83.

41 Cf. les thèses suivantes: Claude L. Shaver, The Delsarte System of Expression As Seen Through the Notes of Steele Mackaye, Ph. D. Dissertation, University of Winsconsin) ; Leslie Carol Schreiber , Movement Training for the Actor : Laying the Foundation in Movement Principles, Ph.D. dissertation, University of Minnesota ; Janis Dawn Clarke, The Influence..., op. cit. ; David Tabish, Kinesthetic Engagement Technique: Theories and Practices for Training the Actor, Ph.D. dissertation, University of Pittsburgh, 1995. sus de base élaborés par Delsarte (on prétend ainsi souvent décontracter le cou en lui faisant faire des rotations, plutôt qu'en laissant jouer le poids de la tête).

De plus, l'insistance de Delsarte sur le rôle moteur et émotionnel du haut du torse et sur la qualité du mouvement associée à la traduction d'un « mouvement intérieur ", restent d'une grande actualité pour une pratique dansée ou pour un regard critique sur les créations chorégraphiques d'aujourd'hui, qui tendent parfois à se rapprocher de pratiques sportives (comme le revendique le chorégraphe suisse Gilles Jobin), et dans lesquelles le bassin est devenu l'élément moteur, au détriment du haut du buste (c'est le cas, par exemple, dans la technique issue de Merce Cunningham).

Enfin, de manière beaucoup plus large, le phénomène américain de Delsartemania, à la fin du XIXe siècle, a montré qu'une pratique corporelle et vocale fondée sur les enseignements de Delsarte, pouvait toucher un large public, au-delà des milieux artistiques. Le travail de Delsarte, qui fut parfois dénommé « yoga occidental ${ }^{42}$, est à la fois exigeant car demandant attention et durée, et simple d'accès car ne visant pas la prouesse technique et prenant en compte des réalités de la personne. II s'apparente, sur bien des points, à certaines des techniques venues d'Asie, comme le yoga ou les arts martiaux, à la différence près qu'il n'a de visée ni martiale ni directement méditative, même s'il est fondé sur l'idée que tout travail du corps a des incidences au niveau intérieur. Ainsi, le travail de l'équilibre corporel, central dans la pratique delsartienne, aurait-il une incidence directe sur l'équilibre psychique - nous pourrions alors parler d'équilibre psy-

42 Cf. annonce publicitaire pour le travail de Hellès-Abilea, in Le Figaro littéraire du 06/01/1962, p. 12. 
chomoteur. Ce travail a aussi la particularité, vis-à-vis des pratiques que nous venons d'évoquer, d'avoir ses référents dans la culture occidentale. Très éloigné de l'idée de gymnastique (dans le sens d'une pratique formelle et mécanique du corps), l'entraînement corporel inclus dans la méthode de Delsarte, reste potentiellement une offre sur un marché du travail du corps et du développement personnel, en pleine expansion dans les sociétés occidentales.

\section{- Les enseignements expressifs de Del- sarte annoncent l'éducation somatique}

Profondément, l'entrainement proposé par la méthode de Delsarte est plus qu'un travail purement corporel : il associe intimement la conscience de ce qui se passe en soi durant les phénomènes expressifs, et en particulier lors des mouvements, le geste étant vu comme la traduction d'un mouvement intime, psychique. II s'agirait alors de prendre conscience de cette relation entre le mouvement et son origine. C'est pourquoi nous avons choisi de parler d'approche « somatique-expressive » concernant Delsarte. Placer l'ensemble des enseignements delsartiens centrés sur le geste sous le terme de "somatique expressif », plutôt que de « corporel expressif » a une vraie portée historique : celle de placer Delsarte comme l'un des inspirateurs de ce qui est aujourd'hui appelé l'éducation somatique. ${ }^{43}$

II ne suffit pas de rappeler, dans cette perspective, que les enseignements de Delsarte ont fait partie de la formation de celui qui est

43 Cf. Franck Waille, La méthode somatique expressive de François Delsarte. Histoire, esthétique, anthropologie : de la neurophysiologie à la métaphysique, Montpellier, L'Entretemps, 2016. considéré comme l'initiateur de l'éducation somatique au XXe siècle, Frederick Matthias Alexander, ou de celle d'autres ayant poursuivi cette démarche, comme Elsa Gindler. Et cela d'autant moins que celui qui a introduit le terme de somatique, Thomas Hanna, ne remonte pas à Delsarte dans la genèse de cette approche, même si les travaux de Delsarte sont mentionnés dans le champs de l'éducation somatique comme participant d'un renouvellement des études culturelles («cultural studies») dans une dynamique pluridisciplinaire. ${ }^{44}$

En choisissant d'appliquer au travail de Delsarte le terme de somatique, nous proposons de voir de manière précise dans ses travaux l'un des éléments de la genèse de l'éducation somatique qui s'est développée au XXe siècle. II s'agit dès lors d'explorer en profondeur les enseignements delsartiens et de montrer en quoi ils peuvent être porteurs des dynamiques qui sont celles de l'éducation somatique, en termes de conception intégrée des différentes dimensions de la personne, et de ce que nous pourrions appeler la pédagogie de l'observation, de l'attention, de la prise de conscience de ce qui se joue en soi dans le mouvement. Son approche met en avant l'expérience et la prise de conscience de ce qui se passe en soi. Plus spécifiquement, elle insiste sur la conscience des phénomènes proprioceptifs, tout comme elle envisage la relation gravitaire et l'orientation de l'attention comme des éléments fondamentaux dans la conduite du mouvement.

Un autre choix est d'utiliser l'expression de « travail somatique expressif » (ou de « méthode somatique expressive »). Cette appellation est inhabituelle dans le domaine de l'éducation so-

44 Cf. Martha Eddy, «A brief history of somatic practices and dance: historical development of the field of somatic education and its relationship to dance ", Journal of Dance \& Somatic Practices, vol. I, no 12009, 2009 ; p. 5. 
matique. Elle rappelle, dans le cas de Delsarte, que tout le travail de préparation qu'il propose et qui s'appuie sur une prise de conscience de processus naturels, a précisément et in fine une visée expressive : il s'agit d'alimenter les capacités créatrices de l'individu, les outils de composition de l'artiste. Si son travail est potentiellement ouvert à toute personne, il a été conçu de manière préférentielle en direction des artistes, et la dimension expressive est chez lui ce qui caractérise sans doute le mieux la capacité d'intégrer les différentes dimensions de la personne. Cette optique expressive n'est pas étrangère aux visées de certaines des pratiques rangées dans le champ de l'éducation somatique, en particulier du travail d'Imgard Bartenieff (collaboratrice de Rudolf Laban), de l'Analyse Fonctionnelle du Corps dans le Mouvement Dansé (AFCMD) initiée par Hubert Godard et Odile Rouquet, ou encore du Body-Mind Centering ${ }^{\circledR}(\mathrm{BMC})$ initié par Bonnie Bainbridge-Cohen. Si l'expression de « travail somatique expressif » est inhabituelle, elle renvoie donc cependant à des perspectives qui sont déjà celles d'une partie de l'éducation somatique contemporaine ${ }^{45}$, où le fonctionnel et l'expressif sont intimement associés.

D'une manière générale, nous avons la conviction que les recherches de Delsarte sont à la fois derrière nous - elles ont permis des dynamiques nouvelles pour les arts de la scène et l'émergence des pratiques d'éducation somatique -, mais qu'elles sont aussi devant nous : nombre de leurs contenus sont encore loin d'être entièrement intégrés, et il y a chez elles

45 Cf. par exemple, P. Chemla \& R. Shusterman, « Conscience soma-esthétique, perception proprioceptive et action ", Communications, 1 (86), 2010, p. 15-24, 2007. une vraie richesse pouvant permettre des explorations passionnantes pour toujours mieux découvrir la merveilleuse organisation expressive qui nous constitue.

Bibliographie

ARNAUD, Angélique (1882). François del Sarte, ses découvertes en esthétique, sa science, sa méthode, précédé de détails sur sa vie, sa famille, ses relations, son caractère, Paris, Ch. Delagrave.

DELAUMOSNE, Abbé (1874). Pratique de l'art oratoire de Delsarte, Paris, Joseph Albanel.

DELSARTE, François (1865). « Esthétique appliquée - Des sources de l'art ", Conférences de l'Association philotechnique, Victor Masson et fils, Paris, p. 89-139.

DELSARTE, François (1882). All the Literary Remains of François Delsarte, in Delsarte System of Oratory, New York, Edgar S. Werner.

DELSARTE, François (2015). “ Conseil à mes élèves », in Waille, Damour, 2015, p. 296-298.

DELSARTE, François (2016a). « Geste de rejet / Geste de l'ordre et de la réitération », in Waille (2016), p. 476-477.

DELSARTE, François(2016b). « Textes inédits de Delsarte sur le corps », in Waille, 2016, p. 479-484.

GIRAUDET, Alfred (1884/1885). « The Delsarte 
System ", Werner's Voice Magazine VII, no 1, dec./jan., p. 9-10.

GIRAUDET, Alfred (1893). « Trueness in Singing ", in Delsarte System of Oratory, New York, Werner, p. 569-573.

GIRAUDET, Alfred (1895). Mimique, Physionomie et Gestes. Méthode Pratique D'après le système de François Del Sarte pour servir à l'expression des sentiments, Paris, Ancienne Maison Quantin/Librairies-Imprimeries Réunies.

GODARD, Hubert (1990). «À propos des théories sur le mouvement », Marsyas, vol. 16, p. 19-23.

HAMEL, Thomas-Étienne (1906). Cours d'éloquence parlée d'après Delsarte, Québec, Imprimerie de la compagnie de l'Événement.

HARBONNIER-TOPIN, Nicole, \& FERRI, Catherine (2011). Pre-movement, an essential moment for the dancer. Communication presented at the Dance Kinesiology Teachers Group Conference (24-26 june 2011), Brockport, New York (http://www.brockport.edu/ dance/docs/DKTGannouncement.pdf).

PORTE, Alain (1992). François Delsarte, une anthologie, Paris, IPMC.

RANDI, Elena (1993). François Delsarte: le leggi del teatro. II pensiero scenico del precursore della danza moderna, Roma, Bulzoni editore.

RANDI, Elena (1996). Il magistero perduto di Delsarte. Dalla Parigi romantica alla modern dance, Padova, Esedra editrice.

RANDI, Elena, BRUNETTI, Simone (2013). I movimenti dell'anima. François Delsarte fra teatro e danza, Bari, Edizioni di Pagina.

RUYTER, Nancy Lee Chalfa (1979). Reformers and visionaries: the Americanization of the Art of Dance, New York, Dance Horizons.

RUYTER, Nancy Lee Chalfa (1991). «Delsarte, son système aux États-Unis », in François Delsarte, 1811-1871. Sources-Pensée, Châteauvallon, Théâtre National de la Danse et de I'Image, p. 33-37.

RUYTER, Nancy Lee Chalfa (1999). The Cultivation of Body and Mind in Nineteenth-Century American Delsartism, Westport Connecticut/ London, Greenwood Press.

SHAVER, Claude L. (1933). The Delsarte System of Expression As Seen Through the Notes of Steele Mackaye, PhD. Dissertation, University of Winsconsin.

SHAVER, Claude L. (1954). « Steele Mackaye and the Delsartean Tradition ", in Karl Wallace, History of Speech Education in America, New York, Appleton-Centery-Crofts, p. 202-218.

SHAWN, Ted (1974). Every Little Movement, a Book about François Delsarte, the Man and his Philosophy, his Science and Applied Aesthetics, the Application of this Science to the Art of the Dance, the Influence of Delsarte on American Dance, Brooklyn, Dance Horizons [1963].

STEBBINS, Genevieve (1977). Delsarte System of Expression, New York, Dance Horizons 
[1902] (first edition in 1885 with the title Delsarte System of Dramatic Expression).

THOMPSON, Mary S. (1892). "Delsartism in America II: The Relation of Gymnastics to Expression", Werner's Voice Magazine, \# 3, mars, p. 60-61.

WAILLE, Franck (2011a). Corps, arts et spiritualité chez François Delsarte (1811-1871). Des interactions dynamiques, Lille, ANRT [2009] (https://scd-resnum.univ-lyon3.fr/out/ theses/2009_out_waille_f.pdf).

WAILLE, Franck (2011b). Trois décennies de recherche européenne sur François Delsarte, Paris, L'Harmattan.

WAILLE, Franck (2016). La méthode somatique expressive de François Delsarte. Histoire, esthétique, anthropologique: de la neurophysiologique à la métaphysique, Montpellier, L'Entretemps.

WAILLE, Franck, DAMOUR, Christophe (ed.) (2015). François Delsarte, une recherche sans fin, Paris, L'Harmattan.

Recebido: 60/08/2017

Aprovado: 13/02/2018 